\title{
Pheromone Pre-Seeding for the Construction of RFID Antenna Structures using ACO
}

\author{
Gerhard Weis*, Andrew Lewis*, Marcus Randall ${ }^{\ddagger}$ and David Thiel ${ }^{\S}$ \\ *Institute for Integrated and Intelligent Systems \\ Griffith University \\ Queensland, Australia \\ Email: \{g.weis,a.lewis\}@griffith.edu.au \\ ${ }^{\ddagger}$ School of Information Technology \\ Bond University \\ Queensland, Australia \\ Email: mrandall@bond.edu.au \\ $\S$ Centre for Wireless Monitoring and Applications \\ Griffith University \\ Queensland, Australia \\ Email: d.thiel@griffith.edu.au
}

\begin{abstract}
Ant colony optimisation is often used to construct solutions to problems for which it has no a-priori knowledge. However, decision makers and designers often have insight and practical experience concerning what constitutes good and acceptable solutions. In this paper we exploit this knowledge by applying a pre-seeding procedure to the initial pheromone structure. Using the real-world RFID antenna design problem, we show that very good solutions can be produced. Moreover, this technique allows fine tuning of the search procedure.
\end{abstract}

Index Terms-Ant colony optimisation, RFID antennas.

\section{INTRODUCTION}

The automatic identification of items is a real-world problem that appears in a diverse range of applications, including, but not limited to, luggage tagging, warehouse inventory tracking, vehicle identification on user-pay motorways, and potentially products sold in shops (such as supermarkets). One technology used for these purposes is radio frequency identification (RFID). In this approach a receiver attached to an item (known as a tag) is exposed to an electro-magnetic field that generates a response from which the item may be uniquely identified. One of the problems that engineers have encountered is the design of compact antenna structures for the tags since, for their practical application in tracking a wide range of different items, they are often very small. Until recently, engineers would manually derive antenna designs according to their intuition and knowledge of the field. This practice is time consuming and may not yield the best results but, since no analytical solutions are known for deriving antenna performance from an arbitrary structure, some form of empirical approach to design has been necessary.

However, intelligent automated processes can be used in the design and evaluation of such antennas. Moreover, search strategies, based on biological paradigms, are able to efficiently and intelligently search large numbers of configura- tions. When designing RFID antennas, an engineer is confronted by the dual problems of constructing a feasible antenna structure and also evaluating and optimising the antenna structure and performance. With a careful choice of a suitable meta-heuristic, it is possible to successfully accomplish both these tasks. While effective, such approaches can become very computationally intensive, due to the large number of evaluations of antenna structures required. The parallel nature of the algorithms used lend themselves to use of parallel or grid computing resources. As a whole, the problem and its solution become an exemplar of a real-world, eScience application.

Ant Colony Optimisation (ACO) [1] represents a family of powerful optimisers that are mainly constructive in nature, and are used for combinatorial problems. One of the main differentiators to other meta-heuristics is that these techniques build and access a common repository of learned information about the problem. This pheromone structure is updated throughout the search process and will gradually come to reflect characteristics of good solutions. This accumulated wisdom of the ant colony has been gained through blind search processes only. It is possible though to supplement this kind of accumulation with human expertise. To achieve this, we explore different kinds of pheromone initialisation for the realworld problem of the design of RFID antennas.

Artificial ants require their pheromone repositories to have initial values so that the characteristic probabilistic selection equations can work from the first iterations of the algorithm. The two broad ways that this initialisation can be achieved is by using a constant or variable amount of pheromone. In the latter case, the different values generally depend on the cost/goodness of the corresponding component. In a simple example, Dorigo and Gambardella [2] initiated each pheromone matrix element for the travelling salesman problem (TSP) with a small value inversely proportional to the length of the 
edge. Solonon [3], Fenet and Solonon [4] and Lin, Lu, Shyu and Tsai [5] each introduced a preprocessing step in which various solutions within the state space were first sampled (by direct application of local search) and then used to form the set of initial pheromone values. While this was an extra computational load, the ants were generally able to converge on good quality solutions in times smaller than their standard counterparts.

While this paper is concerned also with deriving appropriate initial pheromone values, we are interested in the role that the human expert/designer can play in this process. Few works exist in which people directly interact with meta-heuristic algorithms in order to influence their operation. One such example is by Hettenhausen, Lewis and Mostaghim [6] using particle swarm optimisation (PSO). In this work a human expert at various intervals within the search process selected a subset of solutions that replaced the social component of the swarm. Solutions having been chosen intuitively, rather than necessarily algorithmically, this interactive version of particle swarm optimisation was able to produce an attainment surface focussed on a specific area of interest more efficiently and effectively than a standard PSO implementation. The work reported in this paper seeks to implement an approach that allows a similar ability for a decision maker to intervene in the optimisation process, guiding the search towards areas of interest and accelerating the acquisition of preferred solutions.

The remainder of the paper is organised as follows. Section II discusses the RFID antenna design problem in context with ACO algorithms. Section III examines some approaches to pre-seeding the pheromone repository with known good solutions and characteristics of good solutions. Sections IV and $\mathrm{V}$ give the experimental results and conclusions respectively.

\section{ACO Applied to CONSTRUCTING RFID ANTENNAS}

The reader is referred to Lewis, Randall, Galehdar, Thiel and Weis [9] for an in-depth description of how ants construct meander lines and the application of the local search backbite operator. However, it is instructive to note that by use of ACO, both the problem of constructing a trial antenna element, and optimising the resulting structure are accomplished in a single, unified process. It was this ready, almost natural, applicability of ACO to this specific problem that initially led the authors to explore the use of this particular metaheuristic in preference to other methods. To devise a method by which the construction of a generalised meander line can be parametrically described, and incrementally altered, is not a simple task. By limiting the structure to a simplified, prescribed form, there have been some studies of simple RFID antennas [13], [14] using genetic algorithms (GA). Another approach with greater generality, but a radically different approach to the antenna structure, used a GA to optimise a patch antenna [15]. The approach proposed by the authors has recently gathered interest, with derivative work beginning to appear [16]. However, it can still be said that automated approaches to RFID antenna design optimisation have had limited investigation.

One of the variants of ACO is named Ant Colony System (ACS) and is used as the basis of this work and previous RFID based research [7]-[11]. The canonical ACS algorithm has been modified for the problem of creating meander line RFID antennas. Figure 1(a) shows a $5 \times 5$ grid on which the ACS algorithm operates, with 1(b) giving a possible solution (commonly referred to as the plough). The solution is then mirrored on the other half of the antenna, with a connecting $1 \mathrm{~mm}$ bridge. Each half area contains a grid of $n \times n$ points. Regardless of the value of $n$, the physical grid size remains constant at $25 \mathrm{~mm}$.

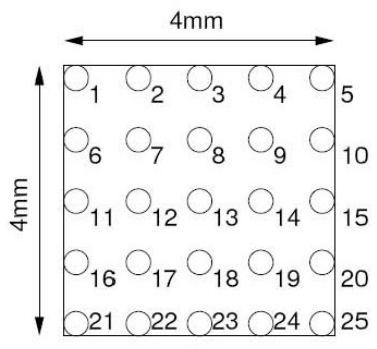

(a)

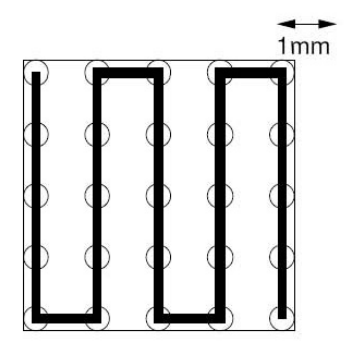

(b)
Fig. 1: (a) defines the grid and numbering system and (b) shows a possible meander line antenna. The latter is referred to as a "plough" structure.

Each ant constructs a path of maximum length. An ant may terminate its path either when it has run out of grid points or it becomes trapped between points it has already visited. As is evident from the above diagram, each point is connected to at most four others. In the single objective version of the problem, the aim is to maximise the efficiency of the antenna. This is a form of black box optimisation as this characteristic is evaluated by the NEC [12] software suite.

An extension to the single objective problem of optimising antenna efficiency is to enable it to optimise multiple objectives such as resonant frequency of operation. Formally, we are interested in maximising antenna efficiency, $\eta$, and minimising the resonant frequency, $f_{0}$. Having multiple objectives means that rather than a single best solution being produced, a tradeoff or attainment surface will be derived instead. It is up to the antenna designer to choose the antenna, or set thereof, that will best meet their needs. For results of the two-objective problem described, see Lewis et al. [8].

Our multi-objective implementation is simple and requires few computational resources. To determine whether one solution is more attractive than another, a domination relation is used. For solution vectors $\overrightarrow{x_{1}}$ and $\overrightarrow{x_{2}}$, when the following conditions are met:

- $\overrightarrow{x_{1}}$ is at least as good as $\overrightarrow{x_{2}}$ for all objectives, and

- $\overrightarrow{x_{1}}$ is strictly better than $\overrightarrow{x_{2}}$ for at least one objectives 
1: A colony of ants begin construction of the walks (antennas) on a Cartesian grid of specified size

2: while each ant has not completed construction of an antenna do

3: for each ant, from a starting node on the edge of the grid do

4: $\quad$ Add the next node to a directed graph, according to the probabilistic selection rules

5: $\quad$ if the ant cannot find a feasible next node then

6: terminate the ant for this iteration

7: $\quad$ end if

8: $\quad$ end for

9: Update the local pheromone data for all ants

10: end while

11: Apply the backbite local search operator to degree three for each solution/antenna

12: Determine if any of the colony's solutions should be added to the Archive

13: For those solutions entering the Archive, update the global pheromone

14: end

Fig. 2: A single iteration of the multi-objective ACS algorithm.

then $\overrightarrow{x_{1}}$ is said to "dominate" $\overrightarrow{x_{2}}$ (denoted $\overrightarrow{x_{1}} \prec \overrightarrow{x_{2}}$ ). In the case where $\overrightarrow{x_{1}}$ and $\overrightarrow{x_{2}}$ dominate other solution vectors but not each other they are deemed mutually equal and become part of the attainment surface. This set of solutions reflects the trade-off surfaces between the different objectives and is kept in a data structure referred to as the Archive. If the attainment surface is the best possible then it is said to be the Pareto-optimal set.

Figure 2 gives an overall mechanical description of the multi-objective ACS implementation.

\section{Pheromone Pre-SEEding}

Many traditionally-designed RFID antenna structures follow the form of the "plough", as shown in Figure 1 (see, for example, Marrocco [13]). However, the structures identified as approaching optimal performance in the previously described multi-objective studies consistently took the form of a "spiral" (see, for example, Figure 3). To determine the relative performance of the two types of structures, it would be advantageous to explore structures that were a variation on the "plough" in terms of their performance. To this end we attempted to encourage ants in the computational experiments to construct such structures by pre-seeding the pheromone matrix along the edges forming the "plough" antenna prior to starting Algorithm 2. To do this, the values of preferred edges were, in the first instance, initialised to values similar to those found at the completion of a normal iteration of the algorithm. The results of this investigation, and the further computational experiments they inspired, are described in the following section.

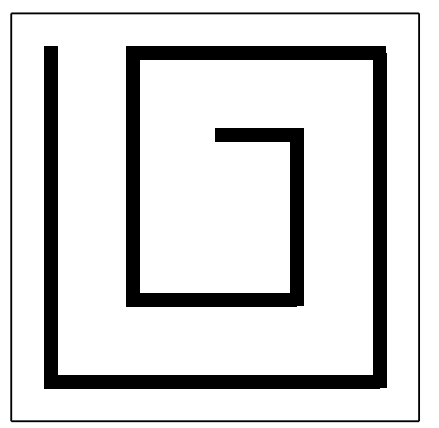

Fig. 3: $5 \times 5$ spiral antenna. Resonant frequency, $f=570 \mathrm{MHz}$, efficiency, $e=92.8 \%$.

\section{COMPUTATIONAL EXPERIMENTS}

The aim of the following experiments is to gauge whether our method of pre-seeding helps to improve antenna structures and allow ants further opportunity to refine existing good designs. As previously mentioned, the authors have gained numerous insights into the nature of good meander line RFID structures through their previous work [7]-[11]. One of the main results was that, regardless of the different types of objectives measures, the ants were robust enough to produce efficient antennas. The efficient designs discovered generally had as their basis plough and spiral formations. Therefore, the experiments in this paper used ant colony pheromone matrices pre-seeded with both structures. As a control measure, the ant colony implementation was also run without pre-seeding.

Initial testing showed that it is necessary to start all the ants from one point on the grid, at the "start" of the preferred path. If this is not done, they will not follow the pre-seeded path in the early iterations of the algorithm, thus losing any potential advantage from using the heuristic knowledge available through pre-seeding. In the previous work by the authors, ants were scattered randomly on the edge of the grid before construction commenced [10].

The details of the size of antennas and the various parameters are follows. The grid size was $25 \mathrm{~mm}$, wire size was 1 $\mathrm{mm}$, and a $6 \mathrm{~mm}$ gap was used between half antennas when joined to form a full dipole antenna. Grid resolutions were tested from $5 \times 5$ up to $10 \times 10$. Three different runs were performed at each grid size, with varying degrees of algorithm "greediness". Each search was permitted 1000 iterations. From experience, this was sufficient to allow adequate convergence of the attainment surface. The objectives were to maximise antenna efficiency, and minimise resonant frequency, which generally has the effect of increasing antenna read distance and maintaining the smallest possible size in terms of wavelength.

Pre-seeding a known good path with a fixed amount of pheromone did not result in any changes in results or algorithm performance. The cause of this is the local pheromone update operation, which imposes an implicit minimum and maximum on the pheromone values. In general such a minimum and maximum value helps the ants in exploring the solution space and prevents their stagnation in local minima. With the 
local pheromone update, pre-seeded pheromones levels were reduced to normal values after 5 to 10 iterations, and after that the ants behaved as without pre-seeding. In case of preseeding, it is important to keep the ants "close" to the preseeded structure. Therefore, it is necessary to alter the ACO algorithm. For this experiment the local pheromone update was removed, in order to slow down the pheromone decay on the pre-seeded tracks. Additionally, it was necessary to change the initial ant placement strategy. To take full advantage of preseeding it is necessary to constrain the ants to start at the point on the grid from which the pre-seeded structure starts.

The amount of pheromone to pre-seed has been chosen by experience from previous experiments. For "unguided" ants (those without pre-seeding), the pheromone matrix had values between $\sim 0$ and $\sim 25$. For the experiments reported in this paper, values of $25,50,75$ and 100 have been chosen. While the pre-seeding effect is less apparent with low values; with values that are too high the ants do not explore the solution space at all.

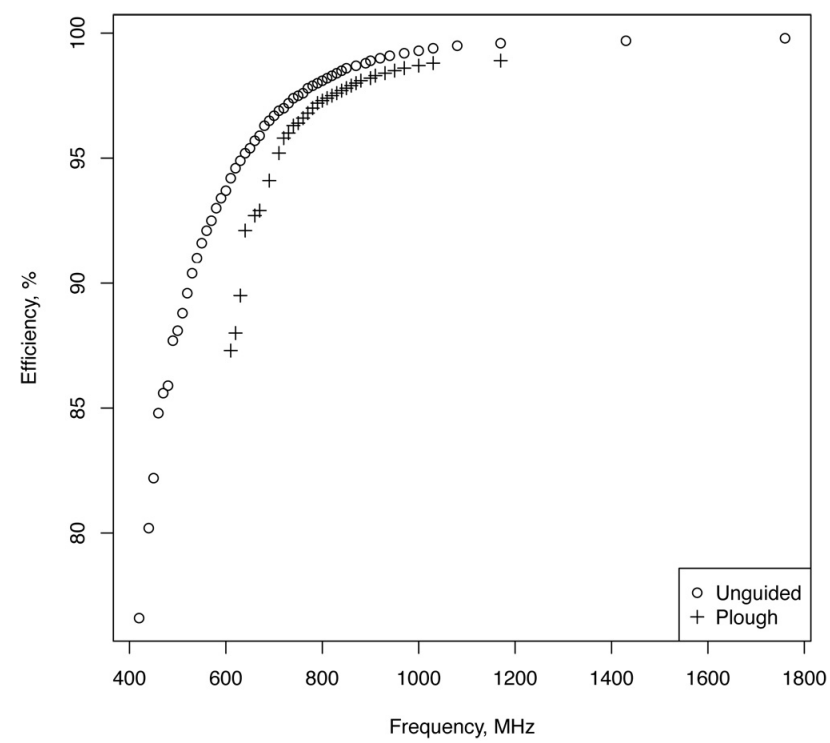

Fig. 4: $10 \times 10$ attainment surface for unguided ants and preseed with plough.

Figure 4 shows two attainment surfaces for antennas generated on a $10 \times 10$ grid. The set of points marked "o" that appear to the left and above in the figure is generated by "unguided" ants and the set of points marked "+", lower and to the right, from runs for which the pheromone matrix has been pre-seeded with a plough structure. For the preseeded attainment surface, the ants generated structures that have shapes characteristic of the "plough" form, as expected. It would appear that pre-seeding the pheromone matrix has successfully guided the ants to search a particular region of the parameter space. Unfortunately these structures generally have lower efficiency and higher minimum resonant frequencies, making them less preferrable than the "spiral-shaped" struc- tures. In Figure 4, comparing pre-seeded and unguided results at some given level of efficiency, it can be seen that unguided searches have yielded antennas with lower resonant frequency. Likewise, comparing results at some desired frequency, the "plough-shaped" antennas give significantly lower efficiency. Experiments with lower pheromone values for pre-seeding have shown that these sub-optimal, "plough-like" structures will be replaced rapidly by "spiral-shaped" structures, as the latter are generally "better" in terms of the given objectives.

Figure 5 shows an example solution from each attainment surface. Figure 5a clearly shows that, when ants are allowed to freely search the solution space, the resulting antenna structures are generally "spiral-shaped". However, in Figure 5b it may be seen that by using pre-seeding it has been possible to generate a range of structures that have, to some greater or lesser extent, some "plough-like" segments.

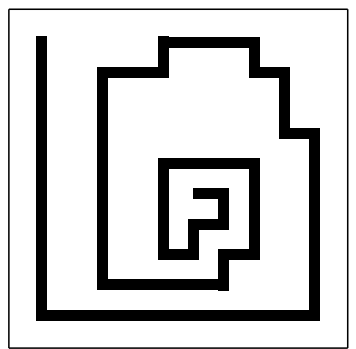

(a)

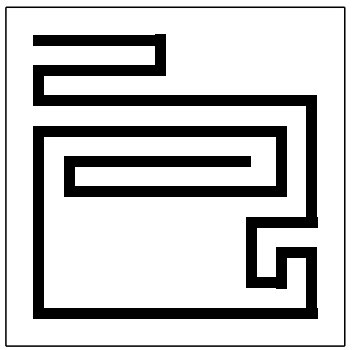

(b)
Fig. 5: Two example solutions from "unguided vs plough" experiment. (a) from "unguided" with $f=560 \mathrm{MHz}, e=$ $92.1 \%$ and (b) from "plough pre-seed" with $f=640 \mathrm{MHz}$, $e=92.1 \%$.

In addition to pre-seeding with conceptual structures about which more information was desired, another experiment was performed in which the pheromone matrix was pre-seeded with a known good structure from an earlier experiment. The aim is to provide a good starting point for further exploration of parameter space. A known good structure that had a low resonant frequency of $f=430 \mathrm{MHz}$, obtained from an unguided run of the experiment, was used to pre-seed the pheromone matrix, as previously described. Figure 6 compares the same "unguided" attainment surface from Figure 4 with an attainment surface achieved by pre-seeding with the known good structure. While the results are almost identical, preseeding enabled the ants to find a number of additional solutions in the lower frequency area.

Besides it being possible to "guide" the ants to certain area in the solution space with pre-seeding, around both good or sub-optimal structures, there are other benefits. As described in earlier work [10], a cache is maintained throughout experimental runs to hold objective values for the various structures tested. When ants construct solutions that have already been evaluated, the results are drawn from the cache, avoiding redundant re-evaluation. The effect is that only unique solutions need be evaluated, so this is a measure of the computational cost of an optimisation experiment. For $5 \times 5$ antennas, un- 


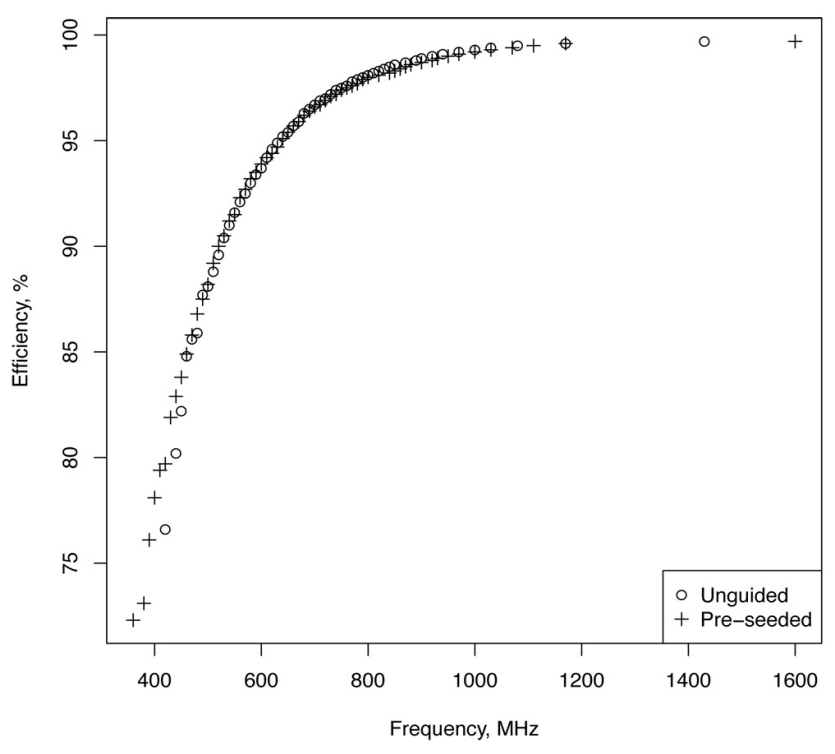

Fig. 6: $10 \times 10$ attainment surface for unguided ants and preseeded with a known good structure.

der different conditions the ants produced markedly different numbers of unique solutions to be evaluated. The following, indicative values were found for differing conditions:

(a) No pre-seeding, using local pheromone update, and initially placing ants randomly around the border, yielded $\sim 16000$ unique solutions.

(b) Initially placing the ants only on the starting point of a preseeded structure, reduced this number to $\sim 3000$ unique solutions

(c) An experiment omitting the local pheromone update step generated only $\sim 1900$ unique solutions.

(d) Pre-seeding the pheromone matrix with a known good solution, reduced the computational effort to $\sim 1400$ unique solutions.

For each of the different cases there was almost no difference in the resulting attainment surface. These results suggest that a few simple measures can radically reduce the computational cost of optimising antenna structures. Finer grid resolutions, with resulting much larger solution spaces, generate many more unique structures for evaluation, so these modifications can be expected to be even more attractive for the larger grid sizes. For comparison, for the last three cases above, the corresponding number of unique solutions tested for a $10 \times 10$ experiment were:

(b) (fixed starting point) $\sim 81000$ unique solutions

(c) (no local pheromone update) $\sim 54000$ unique solutions

(d) (pre-seed with good solution) $\sim 44000$ unique solutions

Pre-seeding with a known good structure appeared to markedly improve algorithm performance. This suggests that it might be possible to use implicit heuristic knowledge obtained from one optimisation experiment to aid in performing more complex optimisation with a greater number of objectives.
To test this hypothesis, a new objective of minimal track width was added to the experiment. The track width between grid points need not be uniform within an antenna, but may vary. The main advantage of smaller track width is a reduced volume of the antenna conducting material, with the potential for reducing negative environmental impact as items "tagged" with RFID chips enter the waste stream. In previous studies, the track width was a uniform $1 \mathrm{~mm}$. In the current work, ants were allowed to choose widths from the set $\{0.25,0.5,0.75,1$ $\mathrm{mm}\}$. An additional pheromone matrix was used to facilitate the colony's ability to learn about this aspect of the problem.

The algorithm to find a path on the grid was left unchanged. To determine wire thickness on each edge, an additional pheromone matrix (in this case a one dimensional list holding one pheromone amount for each possible thickness value) was attached to every possible edge. After the ants had chosen a specific edge during a construction step, they additionally chose a wire width from the attached matrix. The mechanism for choosing a wire width was the same as for choosing the next edge, and the pheromone update and decay was exactly the same as for path finding. However, it should be noted that, by using separate pheromone matrices, the choice of wire width has no influence on the choice of path.

Figure 7 shows two views of the 3-dimensional attainment surface for a $10 \times 10$, three-objective problem, pre-seeded with a known "good" structure from the two-objective experiments. A number of observation may be made:

- There is a convergence of solutions at the "high frequency", "high efficiency" end of the spectrum. These solutions also appear to be of uniformly lower volume of conductor. This could be expected, as antennas of high resonant frequency also tend often to be quite short; they will, therefore, naturally have a lower volume conductor.

- There is an increasing diversity of solutions with lower resonant frequency. The greater potential solution space allowed by antennas constructed from a greater number of edges will likely contain a large number of diverse designs.

- Within the diversity of solutions with lower resonant frequencies, the optimisation procedure has been able to find a number of structures with reduced conductor volume, though there tends to be a correlation with a slight reduction in efficiency. This could be related to the resistance of the antenna segments.

- There remain only a very few solutions at the extremely low resonant frequencies. While the search space may offer many, slightly different structural configurations as the length of the antenna element is increased, there appears little, measurable difference in their performance. Figure 7, it may be noted, does not display the variety of solutions attainable or tested, but only those that are determined to be Pareto-optimal within applied tolerances.

- There are also fewer solutions at the limit of high frequency, both discovered and, in fact, feasible. These antenna elements tend to be very short - there is a general, inverse relationship between antenna length and 
operating frequency - and short elements, made up of fewer segments, allow fewer variations in structure.

Figure 8 shows an example solution for the three-objective optimisation problem. A significant reduction in conductor volume has been achieved, but the structure is quite complex in shape. It remains, basically, a spiral-shaped structure, as guided by the pre-seeding, but the changes in conductor thickness are beyond any current analytical description.

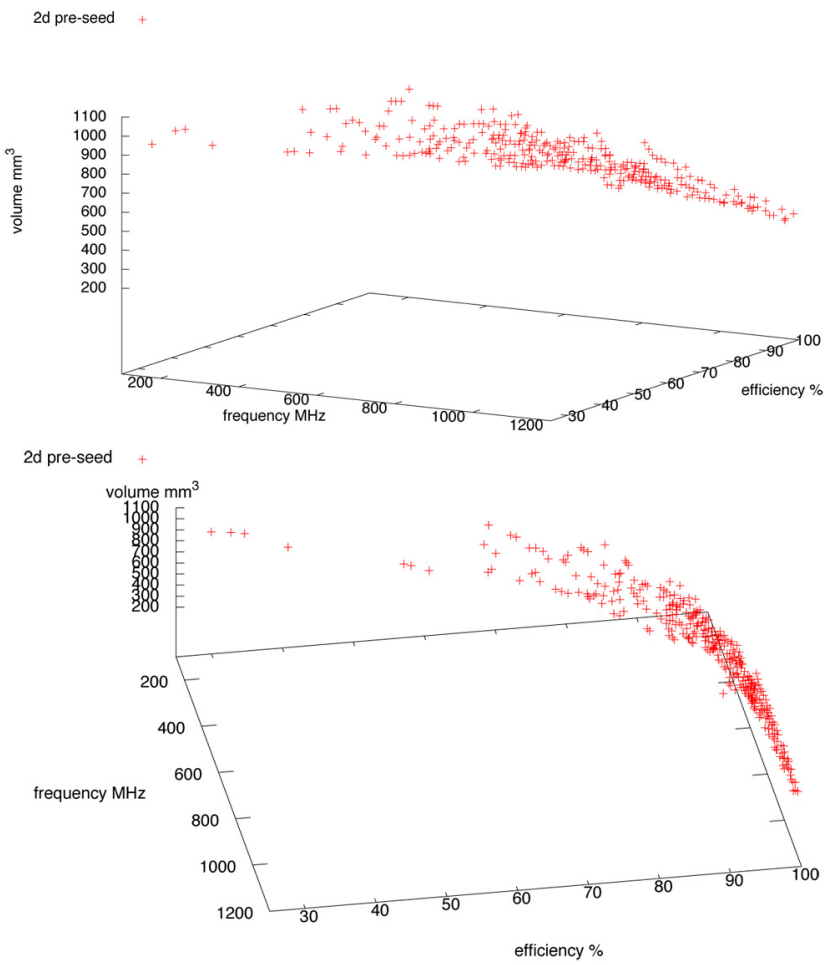

Fig. 7: $10 \times 10$ attainment surface for pre-seed with a known good two-objective structure.

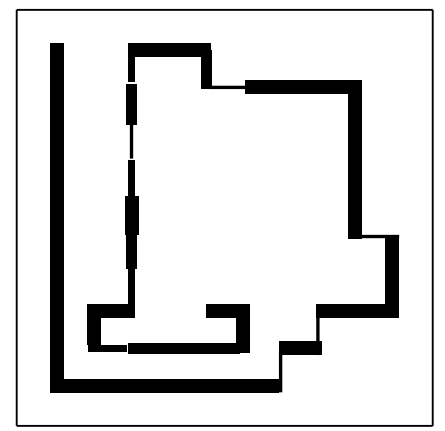

Fig. 8: $10 \times 10$ example solution from three-objective preseeding experiment. $f=490 \mathrm{MHz}, e=82.9 \%$, volume, $v=$ $626.55 \mathrm{~mm}^{3}$.

In summary, the main findings from the pre-seeding experiments are as follows:

- Pre-seeding allowed the exploration of a number of plough-like structures, despite their sub-optimal performance. In an unguided search, these structures are rapidly eliminated.

- These searches of a subset of solution space are achieved with significantly fewer function evaluations than an unguided search.

- If the pre-seeding pheromone value is too low, ants rapidly revert to a more optimal structure (i.e., in the experiments shown, the spiral). If the value is set too high, they will not sufficiently explore and refine the preseeded structure.

- Pre-seeding with a known good structure allows the ants to explore the solution space adequately with significantly reduced numbers of function evaluations, compared to the number required for an unguided search.

- Using pre-seeding techniques, it is possible to convey heuristic knowledge from a simpler optimisation study to a more complex investigation with a greater number of objectives.

\section{Conclusions}

Supplementing the essentially blind search characteristics of meta-heuristic optimisers with human expertise is a novel way of increasing the efficiency of the problem solving process. In this paper, we have used our accumulated experience in the field of RFID antenna design to provide ACO with such a head start. This was achieved by seeding the pheromone matrix with initial values that corresponded to known good antennas. Apart from decreasing the time taken to achieve highly efficient designs, we also found that this knowledge could be extended to more complex forms of optimisation (such as the addition of a new objective).

The next stage in this research is to test the validity of transferring knowledge of antenna structure across changes in grid resolution, by pre-seeding the pheromone matrix with structural elements from a coarse $($ e.g. $5 \times 5)$ grid to a finer $(10 \times 10)$ grid. This would allow the ants more scope for fine-grain exploration, while retaining elements of previous good solutions and potentially reducing the computational load. It will also be interesting to see the effect of this technique on the extended problem, briefly considered here, of constructing antenna elements with desired performance characteristics but reduced material usage and potentially less negative environmental impact.

\section{REFERENCES}

[1] M. Dorigo and G. Di Caro. The ant colony optimization metaheuristic. In D. Corne, M. Dorigo, and F. Glover, editors, New Ideas in Optimization, pages 11-32. McGraw-Hill, London, 1999.

[2] M. Dorigo and L. Gambardella. Ant colony system: A cooperative learning approach to the traveling salesman problem. IEEE Transactions on Evolutionary Computation, 1:53-66, 1997.

[3] C. Solnon. Boosting ACO with a preprocessing step. In Applications of Evolutionary Computing, Proceedings of EvoWorkshops2002: EvoCOP EvoIASP, EvoSTim, pages 161-170. Springer-Verlag, 2002.

[4] S. Fenet and C. Solnon. Searching for maximum cliques with ant colony optimization. In Proceedings of EvoWorkshops 2003: EvoCOP, EvoIASP, EvoSTim, volume 2611 of Lecture Notes in Computer Science, pages 236-245. Springer-Verlag, 2003 
[5] B. Lin, C. Lu, S. Shyu, and Tsai C. Development of new features of ant colony optimization for flowshop scheduling. International Journal of Production Economics, 112(2):742-755, 2008.

[6] J. Hettenhausen, A. Lewis, and S. Mostaghim. Interactive multiobjective particle swarm optimisation with heatmap visualisation based user interface. Engineering Optimization, 42:119-139, 2010.

[7] A. Galehdar, D. Thiel, A. Lewis, and M. Randall. Multiobjective optimization for small meander wire dipole antennas in a fixed area using ant colony system. International Journal of $R F$ and Microwave Computer-Aided Engineering, 19:592-597, 2009.

[8] A. Lewis, G. Weis, M. Randall, A. Galehdar, and D. Thiel. Optimising efficiency and gain of small meander line RFID antennas using ant colony system. In Proceedings of the Congress on Evolutionary Computation, pages 1486-1492, 2009.

[9] A. Lewis, M. Randall, A. Galehdar, D. Thiel, and G. Weis. Using ant colony optimisation to construct meander-line RFID antennas. In A. Lewis, S. Mostaghim, and M. Randall, editors, Biologically-Inspired Optimisation Methods: Parallel Algorithms, Systems and Applications, volume 210 of Studies in Computational Intelligence, pages 189-217. Springer-Verlag, 2009.

[10] M. Randall, A. Lewis, A. Galehdar, and D. Thiel. Using ant colony optimisation to improve the efficiency of small meander line RFID antennas. In Proceedings of the 3rd IEEE International e-Science and Grid Computing Conference, 2007.
[11] G. Weis, A. Lewis, M. Randall, A. Galehdar, and D. Thiel. Local search for ant colony system to improve the efficiency of small meander line RFID antennas. In Proceedings of the Congress on Evolutionary Computation, pages 1708-1713, 2008.

[12] G. Burke, A. Poggio, J. Logan, and J. Rockway. NEC - Numerical electromagnetics code for antennas and scattering. Antennas and Propagation Society International Symposium, 17:147-150, June 1979.

[13] G. Marrocco. Gain-optimized self-resonant meander line antennas for RFID applications. Antennas and Wireless Propagation Letters, 2:302305, 2003.

[14] K.V. Rao, P.V. Nikitin, and S.F. Lam. Antenna design for UHF RFID tags: a review and a practical application. IEEE Transactions on Antennas and Propagation, 53(12):3870-3876, 2005.

[15] G. Kim, and Y-C. Chung. Optimization of UHF RFID tag antennas using a genetic algorithm. In Proceedings of the Antennas and Propagation Society International Symposium, pages 2087-2090, 2006.

[16] V.I Slyusar, and S.Y. Ermolaev. Antenna synthesis based on the ant colony optimization algorithm. In Proceedings of the International Conference on Antenna Theory and Techniques, pages 298-300, 2009. 